\title{
Situación actual y factores que condicionan la lactancia materna en Castilla y León
}

\author{
AM. Sacristán Martín ${ }^{\mathrm{a}}$, JE. Lozano Alonso ${ }^{\mathrm{b}}$, M. Gil Costa ${ }^{\mathrm{b}}$, \\ AT. Vega Alonso ${ }^{b}$, Red Centinela Sanitaria de Castilla y León ${ }^{b}$ \\ ${ }^{a}$ CS Pintor Oliva. Gerencia de Atención Primaria de Palencia. Palencia. España. \\ b Observatorio de Salud Pública. Dirección General de Salud Pública e I+D+i. \\ Consejería de Sanidad. Castilla y León. Valladolid. España.
}

Fecha de publicación en Internet: 23 de febrero de 2011

\section{Resumen}

Objetivos: conocer la prevalencia de inicio de lactancia materna exclusiva en la comunidad de Castilla y León y los factores que influyen en su inicio y duración.

Métodos: estudio descriptivo observacional realizado en la comunidad de Castilla y León (España) de enero a diciembre 2007, en niños entre seis y 12 meses de vida que acudían por cualquier motivo a las consultas de algún miembro del Programa de Pediatría de la Red Centinela Sanitaria de Castilla y León.

Resultados: el porcentaje de mujeres que eligieron lactancia materna exclusiva después del parto fue del $81,2 \%$, el 10,7\% optó por la lactancia mixta y el 8,1\% por la lactancia artificial. El abandono de la lactancia natural fue progresivo mes a mes Ilegando al sexto mes solo el 36,5\% de los niños que la habían iniciado.

Conclusiones: los indices de lactancia materna exclusiva, y sobre todo su mantenimiento hasta los seis meses de vida, continúan alejados de los estándares propuestos a nivel mundial. Es imprescindible una mayor implicación en el fomento de la lactancia natural no solo del personal sanitario sino de la sociedad en general.

Palabras clave: Lactancia materna. Prevalencia. Factores asociados.

Current situation and factors conditioning the start of breastfeeding in Castilla y León

\section{Abstract}

Objectives: to describe the prevalence of exclusive breastfeeding in the community of Castilla y León, and to determine the factors that condition its duration.

Methods: descriptive, observational study carried out in Castilla y León (Spain) in children aged 6 to 12 months attending the consultation of any member of the Health Sentinel Network of Castilla y Leon in 2007.

Results: the percentage of women choosing exclusive postpartum breastfeeding was $81.2 \%, 10.7 \%$ mixed feeding and $8.1 \%$ artificial feeding. The drop-out of breastfeeding was progressive month by month and only $36.5 \%$ of children who initiated it reached six months.

Ana Maria Sacristán Martín, joseignacioyana@msn.com.

Los autores declaran no presentar conflictos de intereses en relación con la preparación y publicación de este artículo. 
Conclusions: the rates of exclusive breastfeeding and the maintenance up to the six months of life are still away from the proposed global standards. It is essential to improve the involvement of health personnel and general society in encouraging breastfeeding.

Key words: Breastfeeding. Prevalence. Associated factors.

\section{Introducción}

Tanto el número de madres que inician la lactancia como la duración en el tiempo de la misma han venido sufriendo fluctuaciones debido a la falta de uniformidad en los profesionales sanitarios y a presiones publicitarias que han llevado al fracaso de la lactancia materna.

Tras la Segunda Guerra Mundial, la lactancia materna fue abandonada de manera progresiva, llegando en los años 70 a niveles mínimos en países como EE. UU. Desde entonces se han venido realizando campañas para promover la lactancia materna, que han logrado un aumento progresivo pero sin llegar a los niveles recomendados a nivel mundial. Las tasas bajas y el abandono precoz del amamantamiento acarrean considerables efectos adversos sanitarios, sociales y económicos.

El amamantamiento es la forma natural de alimentación de los lactantes y la leche humana es el alimento mejor adaptado a las necesidades nutricionales del bebé'.

Los beneficios de la lactancia materna son bien conocidos a corto plazo: mejora de la inmunidad, de la función intestinal, bienestar psicológico y establecimiento del apego $0^{2-5}$. A largo plazo está demostrada su influencia en la obesidad ${ }^{6,7}$, en el riesgo de enfermedad cardiovascular en el adulto ${ }^{8,9}$, en la probabilidad de padecer cáncer ${ }^{10,11}$, en ciertas condiciones alérgi$\mathrm{cas}^{12}$, diabetes mellitus ${ }^{13}$, enfermedades inflamatorias ${ }^{14}$ y en el desarrollo neurocognitivo ${ }^{15-17}$.

Los beneficios no solo son para el bebé, sino que también se extienden a la madre. Favorece la pérdida de peso, disminuye el riesgo de anemia, hipertensión y depresión posparto, y a largo plazo contribuye a disminuir el riesgo de osteoporosis y cánceres de mama y de ovario ${ }^{5}$.

A pesar de la uniformidad en el reconocimiento mundial de estos beneficios, las recomendaciones sobre la duración óptima de la lactancia materna exclusiva se encuentran marcadamente divididas. En 2002, durante la 55. a Asamblea Mundial de Salud, se recomendó la lactancia materna exclusiva (LME) durante los primeros seis meses de vida ${ }^{18}$. La Academia Americana de Pediatría (AAP) recomienda retrasar la alimentación complementaria al 
menos hasta los cuatro meses y mantener la lactancia materna exclusiva hasta los seis $^{19}$. La sociedad Europea de Gastroenterología, Hepatología y Nutrición Pediátrica (ESPGHAN) ${ }^{20}$ recomienda que la alimentación complementaria no sea retrasada más allá de las 26 semanas, basándose en recientes estudios en los que se ha observado menor prevalencia de diabetes tipo $1^{21}$, enfermedad celiaca ${ }^{22}$ y alergia al trigo cuando la introducción de la alimentación complementaria, especialmente cereales, se hace de forma gradual mientras se mantiene la lactancia materna.

Existen numerosos factores que influyen tanto en el inicio como en el mantenimiento de la lactancia materna. Entre los factores positivos, se encuentran la edad, la experiencia previa en lactancia, el nivel de educación, haber tomado una decisión sólida previamente al parto, la educación materna sobre los beneficios de la lactancia materna y el ambiente favorable hospitalario y posteriormente en el entorno materno.

Entre los factores desfavorables se encuentran el tabaquismo, la incorporación de la madre al trabajo, las prácticas hospitalarias erróneas, el ofrecimiento precoz de biberones, el parto mediante cesárea, la enfermedad materna o neonatal, la prematuridad y el bajo peso al nacer $^{23-26}$.

\section{Material y métodos}

Durante el año 2007 se estudiaron de forma retrospectiva 804 niños que acudieron a la consulta de los pediatras de la Red Centinela Sanitaria de Castilla León (RCSCyL). Esta red es un sistema específico de información, orientado a la vigilancia en salud pública y la investigación epidemiológica, basado en la colaboración voluntaria y activa de profesionales sanitarios del Sistema de Salud de Castilla y León, los cuales han sido seleccionados de forma aleatoria entre los médicos y pediatras ateniéndose a criterios de representatividad regional de la población, según factores sociales, demográficos y sanitarios. La población cubierta aproximada es de un $5 \%$ de la población total de Castilla y León.

Se incluyeron todos los niños con edad comprendida entre los seis y los 12 meses de edad que acudieron a la consulta por cualquier motivo: revisión del niño sano, consulta médica o administrativa.

Se pasó un cuestionario a todas las madres (anexo 1) y se complementaron los datos con la revisión de las historias clínicas.

Se definió la lactancia materna exclusiva (LME) como la alimentación exclusiva por leche materna; la lactancia mixta (LM) como la alimentación por leche materna y preparados artificiales, inde- 
pendientemente de su proporción; la lactancia artificial (LA) como la alimentación del niño exclusivamente con preparados artificiales; y la lactancia materna total (LMT) como la inclusión de lactancia materna exclusiva y mixta.

En el cuestionario se recogieron datos acerca del bebé: peso al nacer, edad gestacional sexo, estado de salud; datos de la madre: experiencia previa, número de hijos, factores socioculturales; datos del parto: tipo, gemelaridad; y datos de la lactancia: inicio, causas, abandono, tipo, etc.

El peso de los niños se categorizó en $<3000 \mathrm{y} \geq 3000 \mathrm{~g}$, el nivel de estudios se agrupó en dos niveles: madres sin estudios o con estudios primarios y madres con estudios secundarios o superiores. La edad materna se categorizó en $<35$ años $y>35$ años.

Se calcularon las tasas de prevalencia de inicio de lactancia materna exclusiva, su continuación hasta los cuatro y los seis meses de edad, y de lactancia materna total a los seis meses, y se calcularon intervalos de confianza.

Para comparar la asociación de algunos factores estudiados se realizaron test de chi cuadrado, calculando la odds ratio (OR). Para comparar diferentes variables continuas se utilizó el test de la t de Student.
Además, se realizó un análisis de regresión logística donde la variable de respuesta era la lactancia materna exclusiva y las variables independientes la edad materna, el peso del niño al nacimiento, el tipo de parto (eutócico o no), el nivel de estudios de la madre, el trabajo, el tabaquismo, haber recibido educación sobre lactancia, cursos de preparación al parto, conocer grupos de apoyo a la lactancia, recibir la guía de lactancia, haber sido ayudadas en el hospital en el inicio de la lactancia materna y haber recibido regalos en el hospital de las casas comerciales de leche artificial. Se calcularon las OR y se contrastó la falta de asociación de cada factor con el inicio de la lactancia.

El estudio multivariante se repitió con el mantenimiento de la lactancia materna a los seis meses, y con la lactancia materna total.

\section{Resultados}

La edad media de los niños fue de 8,4 meses (desviación estándar [DE]: 2,2 ). El $50,6 \%$ eran niñas y el $47,8 \%$ niños. El peso medio al nacimiento fue de 3201 g (DE: 502,99). Catorce niños provenían de partos gemelares. El 95,2\% de los niños nació en un hospital público y el $3,4 \%$ en uno privado (tabla 1 ).

La edad media de las madres fue de 32,9 años (DE: 5,1). La edad media de las 


\begin{tabular}{|c|c|c|c|c|}
\hline & & & Casos & $\%$ \\
\hline \multirow[t]{14}{*}{ Pacientes } & Total & & 804 & \\
\hline & \multirow[t]{2}{*}{ Edad } & Entre 6 y 9 meses & 576 & $71,6 \%$ \\
\hline & & Mayores de 9 meses & 228 & $28,4 \%$ \\
\hline & \multirow{2}{*}{ Sexo } & Mujeres & 407 & $50,6 \%$ \\
\hline & & Hombres & 384 & $47,8 \%$ \\
\hline & \multirow[t]{4}{*}{ Peso al nacer ( $\mathrm{g}$ ) } & $<2500$ & 53 & $7,1 \%$ \\
\hline & & $2500-2999$ & 155 & $20,6 \%$ \\
\hline & & $3000-3499$ & 353 & $47 \%$ \\
\hline & & $\geq 3500$ & 190 & $25,3 \%$ \\
\hline & \multirow[t]{2}{*}{ Hospital } & Público & 766 & $96,6 \%$ \\
\hline & & Privado & 27 & $3,4 \%$ \\
\hline & \multirow{3}{*}{ Tipo de parto } & Eutócico & 523 & $65,2 \%$ \\
\hline & & Cesárea & 208 & $25,9 \%$ \\
\hline & & Instrumental & 71 & $8,8 \%$ \\
\hline \multirow[t]{11}{*}{ Madres } & \multirow[t]{3}{*}{ Distribución paridad } & Primípara & 432 & $53,9 \%$ \\
\hline & & Secundípara & 302 & $37,9 \%$ \\
\hline & & Tercero o más & 65 & $8,1 \%$ \\
\hline & \multirow[t]{2}{*}{ Experiencia previa } & Lactancia materna en hijos anteriores & 320 & $86,5 \%$ \\
\hline & & No & 50 & $13,5 \%$ \\
\hline & \multirow[t]{2}{*}{ Nivel de estudios } & Sin estudios o primarios & 180 & $22,7 \%$ \\
\hline & & Secundarios o superiores & 608 & $77,2 \%$ \\
\hline & \multirow[t]{2}{*}{ Trabajo fuera de casa } & Sí & 485 & $60,3 \%$ \\
\hline & & No & 319 & $39,7 \%$ \\
\hline & \multirow[t]{2}{*}{ Fumadora habitual } & Sí & 141 & $17,5 \%$ \\
\hline & & No & 663 & $82,5 \%$ \\
\hline
\end{tabular}

madres primíparas fue de 31,6 años (DE: 4,9). En el $53,7 \%$ de los casos, este era su primer hijo, en un $37,9 \%$ el segundo y en el $8,1 \%$ tenían dos o más hijos previos. La mayoría de las mujeres que ya tenían hijos refieren experiencia previa de lactancia materna $(86,5 \%)$, lo que representa el $39,8 \%$ del total de las madres. El $36,4 \%$ tenía estudios superiores, un $40,2 \%$ había terminado los estudios secundarios, el $17,8 \%$ los primarios, un $5,1 \%$ no había completado los estudios primarios. El $60,3 \%$ trabajaba fuera del hogar, el 17,5\% fumaba de forma habitual. El 75,1\% realizó cursos de preparación al parto y el 68,6\% recibió educación para la lactancia materna. El 52,5\% conocía los grupos de apoyo a la lactancia materna y el 68,1\% había recibido la guía para padres del programa de promoción de la lactancia materna. El 70,5\% había recibido apoyo en el hospital para el inicio del amamantamiento. Pese a los esfuerzos por evitar la presión comercial sobre 
la lactancia materna, un 50,2\% de las madres que contestaban aseguraba haber recibido regalos de las casas comerciales de alimentación infantil (tabla 1).

En cuanto al tipo de parto, en el $65 \%$ fue normal, el $26 \%$ precisó cesárea y en un $8,8 \%$ fue necesario emplear instrumental por distocia (tabla 1).

El porcentaje de mujeres que eligieron lactancia materna exclusiva después del parto fue del $82,7 \%$, el $8,6 \%$ lactancia mixta y el $8,6 \%$ lactancia artificial. Entre los motivos que llevaron a las madres a elegir la lactancia materna exclusiva, el $75,7 \%$ adujo como principal motivo para la elección el considerar esta como lo mejor para el niño, el 38,1\% lo consideraba más cómodo, el 31,6\% destacaban el factor económico y un $43,5 \%$ lo hacía por consejo sanitario.

La decisión de lactancia artificial o abandono de la lactancia materna exclusiva en un $28,9 \%$ fue a iniciativa materna, un $21,6 \%$ a causa de consejos sanitarios y un $17,3 \%$ por el entorno de la madre. Entre los motivos que las madres alegaban, el más frecuente fue la hipogalactia $29,7 \%$ seguida de la escasa ganancia de peso $20,5 \%$. La necesidad de incorporarse al trabajo ocupó un tercer lugar, con un $11,8 \%$, y ya mucho menos frecuentes fueron la enfermedad materna 5,1\% o del niño 5,7\%, la incomodi- dad para la madre $2,7 \%$ y motivos estéticos en seis madres $(0,3 \%)$.

La duración media de la lactancia materna exclusiva fue de 3,9 meses (intervalo de confianza del $95 \%$ [IC 95\%]: $3,89-4,09)$ y la de la lactancia materna total de 4,6 meses (IC 95\%: 4,10-4,38).

De los que iniciaron lactancia materna exclusiva, el 64,9\% (IC 95\%: 58,7-70,6) llegó a los cuatro meses y solo el $27,1 \%$ (IC 95\%: 21,8-39,9) llegó a los seis meses con la misma.

Haber amamantado anteriormente favorece el inicio de la lactancia materna exclusiva: el $89,4 \%$ de las madres que ya lo habían hecho salían del hospital con lactancia materna exclusiva frente a solo un $40,0 \%$ de las madres sin experiencia previa en lactancia $(p<0,01)$.

En cuanto a los factores que influyeron en el inicio de la lactancia materna exclusiva (tabla 2), se observó que el peso del bebé al nacer es significativo $(p<0,01)$, los que pesan más de $3000 \mathrm{~g}$ tienen mayor probabilidad de ser amamantados que los que pesan menos de $3000 \mathrm{~g}$ OR: 4,1 (IC 95\%: 1,83-9,36).

El tipo de parto resultó significativo $(p<$ $0,05)$; los niños procedentes de un parto eutócico recibieron lactancia materna exclusiva con mayor frecuencia que los procedentes de partos distócicos o cesáreas, OR: 2,54 (IC 95\%: 1,09-5,93). También 
Tabla 2. Factores que influyen en el inicio de la lactancia materna exclusiva

\begin{tabular}{l|c|c|c}
\hline & OR & IC 95\% & P \\
\hline Peso recién nacido $(\geq 3000 \mathrm{~g}$ vs. $<3000 \mathrm{~g}$ ) & 4,13 & $1,83-9,36$ & $<0,01$ \\
\hline Edad materna & 1,02 & $0,94-1,12$ & 0,53 \\
\hline Tipo de parto (eutócico vs. instrumental o cesárea) & 2,54 & $1,09-5,93$ & $<0,05$ \\
\hline Nivel de estudios (medios/superiores vs. sin estudios/primarios) & 1,48 & $0,52-4,17$ & 0,45 \\
\hline Trabajo (sí vs. no) & 0,28 & $0,10-0,78$ & $<0,05$ \\
Tabaquismo (sí vs. no) & 0,30 & $0,12-0,77$ & $<0,05$ \\
\hline Guía de lactancia & 1,72 & $0,65-4,50$ & 0,26 \\
\hline Curso preparación al parto & 1,33 & $0,23-7,67$ & 0,74 \\
\hline Clases de lactancia materna & 0,73 & $0,13-4,05$ & 0,72 \\
\hline Apoyo en el hospital & 1,36 & $0,58-3,22$ & 0,47 \\
Grupos de apoyo a la lactancia & 0,91 & $0,35-2,40$ & 0,86 \\
\hline Regalos & 1,55 & $0,67-3,55$ & 0,29 \\
\hline IC: intervalo de confianza; OR: odds ratio. & & \\
\hline
\end{tabular}

se encontró significativo el tabaquismo materno $(p<0,01)$, ya que las madres fumadoras tenían menor probabilidad de amamantar a sus hijos, OR: 0,30 (IC 95\%: 0,12-0,77).

El resto de factores que se incluyeron en el análisis multivariante no resultaron significativos. Entre estos factores destaca el nivel de estudios de la madre, que está relacionado con el hábito tabáquico, OR: 2,36 (IC 95\%: 1,33-4,20) y el trabajo fuera del hogar, OR: 3,77 (IC 95\%: 2,25-6,19) aunque no tenga relación con la LME.

Cuando analizamos conjuntamente los mismos factores y su relación con la duración de la lactancia materna exclusiva a los cuatro y a los seis meses, encontra-

Tabla 3. Factores que influyen en el mantenimiento de la lactancia materna exclusiva a los seis meses

\begin{tabular}{l|c|c|c}
\hline & OR & IC 95\% & P \\
\hline Peso recién nacido ( $\geq 3000$ g vs. $<3000 \mathrm{~g}$ ) & 1,09 & $0,37-3,23$ & 0,86 \\
Edad materna & 1,04 & $0,96-1,13$ & 0,24 \\
\hline Tipo de parto (eutócico vs. instrumental o cesárea) & 0,51 & $0,16-1,57$ & 0,24 \\
\hline Nivel de estudios (medios/superiores vs. sin estudios/primarios) & 0,06 & $0,00-0,56$ & $<0,05$ \\
\hline Trabajo (sí vs. no) & 0,54 & $0,23-1,28$ & 0,16 \\
\hline Tabaquismo (sí vs. no) & 0,51 & $0,04-5,24$ & 0,57 \\
Guía de lactancia & 3,37 & $0,34-32,99$ & 0,29 \\
\hline Curso preparación al parto & 0,85 & $0,35-2,00$ & 0,73 \\
\hline Clases lactancia materna & 0,81 & $0,32-2,00$ & 0,65 \\
\hline Apoyo en el hospital & 1,03 & $0,43-2,47$ & 0,99 \\
\hline Grupos de apoyo a la lactancia & 0,82 & $0,34-1,96$ & 0,66 \\
Regalos & 1,45 & $0,62-3,40$ & 0,33 \\
\hline IC: intervalo de confianza; OR: odds ratio. & & \\
\hline
\end{tabular}


Tabla 4. Factores que influyen en el mantenimiento de la lactancia materna total a los seis meses

\begin{tabular}{l|c|c|c}
\hline & OR & IC 95\% & P \\
\hline Peso recién nacido ( $\geq 3000 \mathrm{~g}$ vs. $<3000 \mathrm{~g}$ ) & 1,78 & $0,75-4,23$ & 0,19 \\
\hline Edad materna & 0,98 & $0,91-1,05$ & 0,70 \\
\hline Tipo de parto (eutócico vs. instrumental o cesárea) & 1,00 & $0,40-2,53$ & 0,98 \\
\hline Nivel de estudios (medios/superiores vs. sin estudios/primarios) & 0,70 & $0,33-1,47$ & 0,35 \\
\hline Trabajo (sí vs. no) & 0,24 & $0,08-0,75$ & 0,01 \\
Tabaquismo (sí vs. no) & 1,22 & $0,25-5,94$ & 0,80 \\
\hline Guía de lactancia & 1,04 & $0,22-4,88$ & 0,95 \\
Curso preparación al parto & 0,97 & $0,44-2,13$ & 0,51 \\
Clases lactancia materna & 1,29 & $0,59-2,81$ & 0,94 \\
Apoyo en el hospital & 1,53 & $0,71-3,30$ & 0,45 \\
Grupos de apoyo a la lactancia & 0,74 & $0,35-1,58$ & 0,27 \\
\hline Regalos & 1,43 & $0,71-2,90$ & 0,31 \\
\hline IC: intervalo de confianza; OR: odds ratio. &
\end{tabular}

mos que solo es significativo el hábito tabáquico, OR: 0,28 (IC 95\%: 0,09-0,79) a los cuatro meses y OR: 0,06 (IC 95\%: $0,00-0,56)$ a los seis meses (tabla 3).

En cuanto a la lactancia materna total (ya sea exclusiva o no), el $61,8 \%$ del total de niños (IC 95\%: 55,9-67,4) llegó a los cuatro meses con lactancia materna, y el 38,5\% (IC 95\%: 32,9-44,4) la mantuvo hasta los seis meses.

Igualmente, en el mantenimiento de la lactancia total a los seis meses, el tabaco (tabla 4) aparece como único factor determinante ( $p<0,05$; OR: 0,24; IC 95\%: 0,08-0,75).

\section{Discusión}

La lactancia materna no es solo una forma de alimentar al niño sino que también contribuye al establecimiento del vínculo afectivo entre la madre y el hijo.
En las últimas décadas hemos asistido a un incremento progresivo de la lactancia materna $^{27-29}$.

Los datos de nuestro estudio, con un inicio de la LME del 82,7\%, están de acuerdo con el objetivo de la OMS para 2010 y son similares o discretamente superiores a los reflejados en los últimos estudios realizados en nuestro entorno $0^{30,31} \mathrm{y}$ muy superiores a los publicados recientemente por otros autores ${ }^{32,33}$.

La duración media de la LME (3,9 meses) y la LMT (incluidas exclusiva y mixta) fue ligeramente superior a la última encuesta realizada en el ámbito nacional por García ${ }^{27}$.

Casi el 65\% mantuvo la lactancia materna exclusiva a los cuatro meses, pero solo el $27 \%$ llegó a los seis meses, el $50 \%$ de las madres refería hipogalactia (si bien la hipogalactia verdadera no llega 
a afectar a más del $10 \%$ de las mujeres) o baja ganancia de peso como motivo del abandono de la $L M E$, lo que puede reflejar una introducción temprana de la alimentación complementaria debido a una interpretación errónea de la ganancia ponderal adecuada.

Preocupa que en el $20 \%$ de los casos el consejo sanitario fuera referido como motivo de abandono, lo cual puede deberse a que los niños con lactancia natural tienen una curva de ganancia ponderal distinta a la de los alimentados con lactancia mixta o artificial. Las curvas que mayoritariamente utilizamos en nuestra región ${ }^{34}$ están basadas en población con mucho mayor porcentaje de estos tipos de lactancia que la que tenemos actualmente, por lo que la aparente escasa ganancia ponderal puede ser engañosa y no justificar por sí misma el abandono de la LM. Sería recomendable la utilización de unas tablas basadas en niños lactados de forma exclusiva al pecho como las de la OMS de $2006^{35}$.

También es posible un escaso refuerzo en el entorno para su mantenimiento: inseguridad de la madre para mantener el pecho, de forma que interpreta el llanto o la intranquilidad del niño sistemáticamente como hambre.

A la hora de comparar los distintos estudios, hay que tener en cuenta la distinta metodología empleada y las diferencias de la población estudiada. Por ejemplo, Castilla y León tiene menos población de inmigrantes que otras áreas como Cataluña y la Comunidad Valenciana, donde la tendencia de esta población inicia con mayor frecuencia y tiende a unas lactancias más duraderas ${ }^{36}$.

Si comparamos estos datos con los de un estudio similar realizado en el mismo ámbito poblacional y con la misma metodología de la Red Centinela Sanitaria de Castilla y León en 1998, encontramos una clara mejoría pasando la lactancia materna exclusiva de 7,3\% a los seis meses a un $29,7 \%$ y la lactancia materna total del $21 \%$ al $35,6 \%$ pero siguen siendo niveles inferiores a los estándares recomendados por la OMS.

Los factores relacionados con la lactancia materna son similares a los referidos en otros estudios ${ }^{37,38}$. Ente los factores que favorecen el inicio de la $L M E$, las madres con experiencia previa son las que la inician más pero esta experiencia en nuestro estudio no se ha manifestado en cuanto a la duración tanto de forma exclusiva como mixta.

Como cabía esperar, el peso del niño al nacimiento es crucial para la utilización de la LME. Los partos distócicos también dificultaron la LME. En nuestro estudio, 
el tabaquismo estuvo relacionado con dar a luz a niños de menor tamaño.

La edad media de nuestras madres es mayor, incluso si consideramos solo primíparas (31,6 años), que la que aparece en estudios previos ${ }^{27}$, por lo que los datos no son directamente comparables. Esto refleja el retraso progresivo del inicio de la maternidad que presenta la población española.

A diferencia de otros estudios comentados previamente, en el análisis multivariante de nuestro estudio la formación materna no pareció influir en los hábitos de lactancia.

Si bien es verdad que las madres con mayor formación trabajaban en mayor medida fuera del hogar (el 63,9\% frente a un $32,2 \%$ ) y el trabajo se mostró como un factor limitador en el inicio de la lactancia materna, aunque luego sorprendentemente no llegó a adquirir significación en su duración.

El tabaco se mostró desfavorable tanto para el inicio de la LME como para su mantenimiento. Entristece ver que menos de la mitad de las madres con LME a los cuatro meses la mantiene a los seis.

El $60 \%$ de las madres de nuestro estudio trabaja fuera de casa $y$, aunque solo el $12 \%$ de las madres considera que el trabajo fue el motivo del abandono de la lactancia materna, creemos que una prolongación del permiso materno hasta los seis meses favorecería el mantenimiento de la LME.

Nosotros no hemos encontrado una gran influencia de la educación prenatal, quizás por el alto porcentaje que asiste a la misma y reciben educación sobre lactancia, lo cual hace que las mujeres se decidan mayoritariamente por la lactancia natural; aunque, ello no obstante, los mecanismos de mantenimiento de la misma están fallando.

Tenemos que considerar que la madre lactante se muestra sensible a las presiones del entorno y si en este no está establecida claramente la cultura de la lactancia natural las madres pueden abandonarla demasiado pronto.

\section{Conclusiones}

En nuestra comunidad se ha producido un incremento progresivo del inicio de la lactancia materna exclusiva favorecido por la amplia implantación de los cursos de preparación al parto en los cuales los distintos profesionales sanitarios se implican en la divulgación de los beneficios de la misma.

Todavía nos falta conseguir un mantenimiento óptimo de la lactancia materna, que debería ser exclusiva al menos hasta los cuatro meses, para lo cual es precisa 
la implicación no solo de los profesionales sanitarios, sino también la educación de la población general para crear un ambiente social favorable a la lactancia.

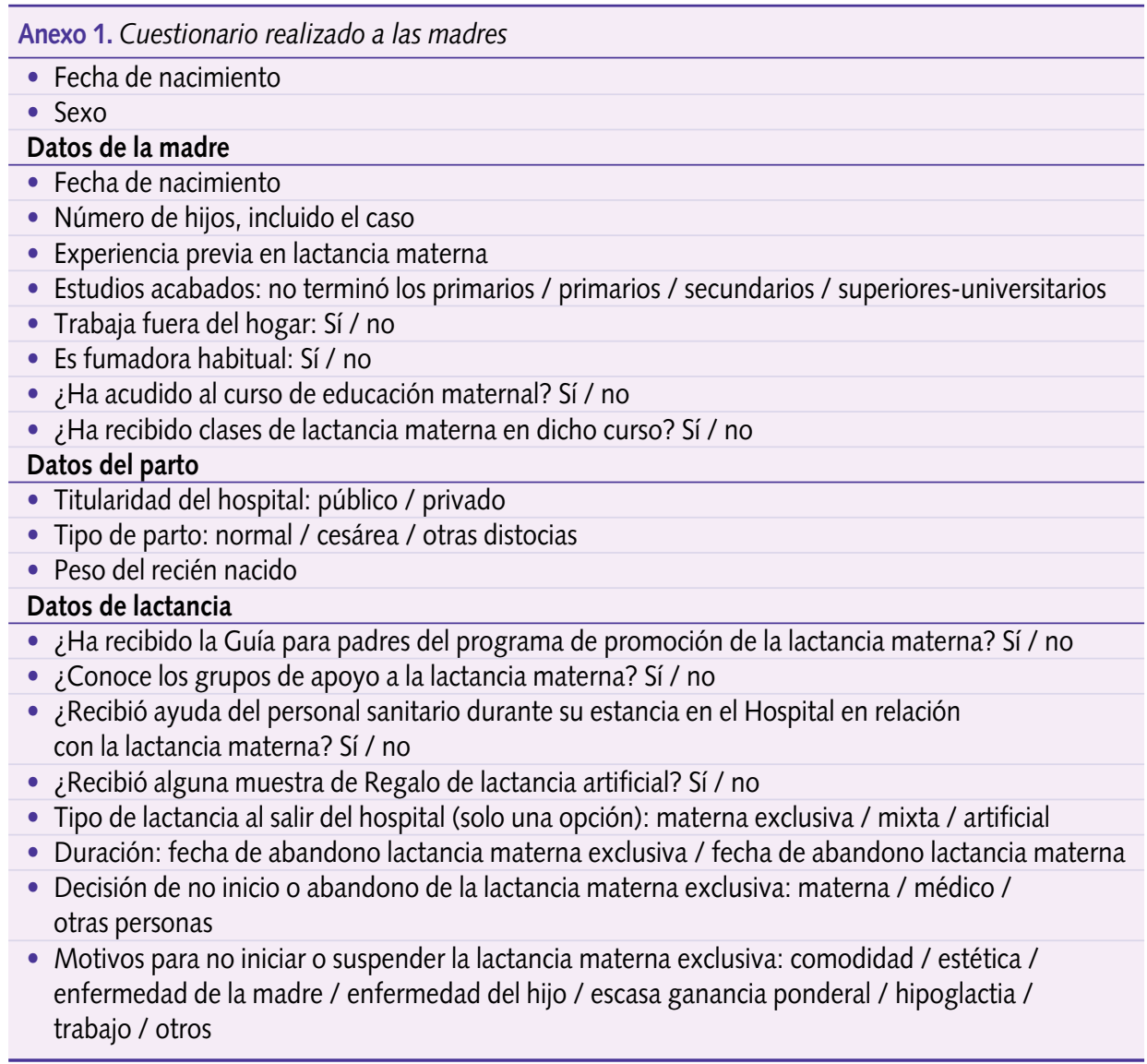

\section{Bibliografía}

1. Cattaneo A, Fallon M, Kewitz G, MikielKostyra K, Robertson A. Alimentación de los lactantes y de los niños pequeños: Normas recomendadas por la Unión Europea EUNUTNET (Red Europea para la Nutrición Pública Saludable: Trabajo en red, monitorización, interven- ción y formación). Comisión Europea (SPC 2003320). 2005-2006 [consultado el 01/05/ 2009]. Disponible en www.burlo.trieste.it/old_ site/Burlo\%20English\%20version/Activities/ research_develop.htm

2. Oddy WH. The impact of breastmilk on infant and child health. Breastfeed Rev. 2002;10:518. 
3. Pardo-Crespo R, Pérez-Iglesias R, Llorca J, Álvarez-Granda $L$, García-Fuentes $M$, Martínez-González MA, et al. Breast-feeding and risk of hospitalization for all causes and fever of unknown origin. Eur J Public Health. 2004; 14:230-4.

4. Department of Health and Human Services, Office on Women's Health. Breastfeeding: HHS Blueprint for Action on Breastfeeding. US Department of Health and Human Services, Washington, DC, 2000.

5. American College of Obstetricians and Gynecologists. Breastfeeding: maternal and infant aspects. ACOG Educational Bulletin 258, American College of Obstetricians and Gynecologists, Washington DC, 2000.

6. Harder $T$, Bergmann $R$, Kallischning $G$, Plagemann $A$. Duration of Breastfeeding and Risk of overweight: A meta-analysis. Am J Epidemiol. 2005;162:397-403.

7. Morán Rodríguez M, Naveiro Rilo JC, Blanco Fernández E, Cabañeros Arias I, Rodríguez Fernández $M$, Peral Casado A. Prevalencia y duración de la lactancia materna. Influencia sobre el peso y la morbilidad. Nutr Hosp. 2009;24:213-17.

8. Rudnicka AR, Owen CG, Strachan DP. The Effect of Breastfeeding on Cardiorespiratory Risk Factors in Adult Life. Pediatrics. 2007;119:e110715.

9. Owen VG, Whincup PH, Kaye SJ, Martin RM, Davey Smith G, Cook DG, et al. Does initial breastfeeding lead to lower blood cholesterol in adult life? A quantitative review of the evidence. Am J Clin Nutr. 2008;88:305-14.

10. Kwan ML, Buffler PA, Abrams B, Kiley VA. Breastfeeding and the risk of childhood leukemia: a meta-analysis. Public Health Rep. 2004;119: 521-35.

11. Ip S, Chung M, Raman $G$, Chew $P$, Magula $N$, DeVine $D$, et al. Breastfeeding and Maternal and Infant Health Outcomes in Developed Countries. Evidence Report/Technology Assessment no. 153. (Prepared by Tufts-New England Medical Center Evidence-based Practice Center, under contract no. 290-02-0022.) AHRQ Publication no. 07-E007. Rockville, MD: Agency for Healthcare Research and Quality; 2007.

12. Gdalevich $M$, Mimouni $D$, Mimouni $M$. Breast-feeding and the risk of bronchial asthma in childhood: a systematic review with metaanalysis of prospective studies. J Pediatr. 2001; 139:261-6.

13. Owen CG, Martin RM, Whincup PH, Smith $G D$, Cook DG. Does breastfeeding influence risk of type 2 diabetes in later life? A quantitative analysis of published evidence. Am J Clin Nutr. 2006;84:1043-54.

14. Koletzko $S$, Sherman P, Corey M, Griffiths $A$, Smith C. Role of infant feeding practices in development of Crohn's disease in childhood. BMJ. 1989:298:1617-8.

15. Sacker A, Quigley MA, Kelly YJ. Breastfeeding and developmental delay: findings from the millennium cohort study. Pediatrics. 2006;118: e682-9.

16. Kramer MS, Aboud F, Mironova E, Vanilovich I, Platt RW, Matush L, et al. Breastfeeding and child cognitive development: new evidence from a large randomized trial. Arch Gen Psychiatry. 2008;65:578-84.

17. Der G, Batty GD, Deary IJ. Effect of breast feeding on intelligence in children: prospective study, sibling pairs analysis, and meta-analysis. BMJ. 2006;333:945.

18. OMS. Nutrición del lactante y del niño pequeño. Estrategia mundial para la alimentación de lactante y del niño pequeño. Informe de la Secretaría. 55. ${ }^{\text {a }}$ Asamblea Mundial de la Salud. Punto 13.10 del orden del día. A55/15. 16 de abril de 2002. 
19. Gartner LM, Morton J, Lawrence RA, Naylor AJ, O'Hare D, Schanler RJ, et al. American Academy of Pediatrics, Section on Breastfeeding. Breastfeeding and the use of human milk. Pediatrics. 2005;115:496-506.

20. Agostoni C, Decsi T, Fewtrell M, Goulet O, Kolacek S, Koletzko B, et al. Complementary feeding: a commentary by the ESPGHAN Committee on Nutrition. J Pediatr Gastroenterol Nutr. 2008;46:99-110.

21. Ziegler $P$, Hanson $C$, Ponza $M$, Novak $T$, Hendricks KJ. Feeding infants and toddlers study: meal and snack intakes of Hispanic and non-Hispanic infants and toddlers. Am Diet Assoc. 2006;106(1S):107-23.

22. Ivarsson A. Epidemic of celiac disease in Swedish children. Acta Pediatr. 2000;89:16571.

23. Suarez $P$, Alonso J, López $A$, Martín $D$, Martínez M. Prevalence and duration of breastfeeding in Asturias. Gac Sanit. 2001;15:104-10.

24. Dubois L, Girard M. Social determinants of initiation, duration and exclusivity of breastfeeding at the population level: the results of the Longitudinal Study of Child Development in Quebec (ELDEQ) 1998-2002). Can J Public Health. 2003;94:300-5.

25. Merten S, Dratva J, Ackermann-Liebrich U. Do baby-friendly hospitals influence breastfeeding duration on a national level? Pediatrics. 2005;116:e702-8.

26. Hernández MT. Epidemiología de la lactancia materna. Prevalencia y tendencias de la lactancia materna en el mundo y en España. En: Lactancia materna: guía para profesionales. Comité de la Lactancia Materna de la AEP. Monografías de la AEP n. ${ }^{\circ}$ 5. Madrid: Ergón; 2004.

27. Martín Calama J. Lactancia materna en España hoy. Bol Pediatr. Asturias, Cantabria, Castilla y León. 1997;37:132-42.
28. García Vera C, Martín Calama J. Lactancia materna en España. Resultado de una encuesta de ámbito estatal. Rev Pediatr Aten Primaria. 2000;2: 373-87.

29. American academy of Pediatrics. Policy Statement. Section on Breastfeeding. Breastfeeding and the Use of Human Milk. Pediatrics. 2005; 115(2).

30. González Oya JL, Valls Durán T, Mosquera González E, Herranz Álvarez M, López Ozores Y, Sanromán Varela MJ, y cols. Estudio sobre la prevalencia de lactancia materna en los centros de salud de Valmiñor. Rev Pediatr Aten Primaria. 2008;10:603-16.

31. González Herrero M, Toledano Bueno J. La lactancia materna en nuestro medio: análisis de la situación. Acta Pediatr Esp. 2007;65:123-5.

32. Romagosa Albacar $C$, Sague Cateura $M$, Garatea Zubieta A, Burgués Burgués $M$. Lactancia materna; factores condicionantes. Rev Enfermería. 2003;26:73-6.

33. Rodicio García MM, Abadi Abadi A, Silveira Cancela $M$, Rodríguez Sáez $M J$, Andrés Andrés $A$, Vázquez Caruncho $M$. Trends and course of breastfeeding in the north of Lugo, Spain. Acta Pediatr Esp. 2007;65:6-11.

34. Sobradillo B, Aguirre A, Aresti U, Bilbao A, Fernández-Ramos $C$, Lizárraga $A$, y cols. Curvas y tablas de crecimiento (estudios longitudinal y transversal). Instituto de Investigación sobre Crecimiento y Desarrollo. Bilbao: Fundación Faustino Orbegozo Eizaguirre; 2004.

35. WHO. Child Growth Standards World Health Organization, Geneva, 2006 [consultado el 16/05/2010]. Disponible en www.who.int/ nutrition/media_page/en/

36. Raventós Canet A, Crespo García A, Villa Vázquez $\mathrm{S}$. Prevalencia de la lactancia materna en el Área Básica de Salud Sant Feliu-2 (Barcelona). Rev Pediatr Aten Primaria. 2006;8:409-20. 
37. Fidalgo Álvarez I, López Pacios $D$, Jiménez Rodríguez M, González Martínez A, Costales Álvarez $A$, Blanco Franco MP, y cols. Factores relacionados con la lactancia materna. Rev Pediatr Aten Primaria. 2002;4:579-91.
38. Barriuso Lapresa LM. Estudio multivariante sobre la prevalencia de la lactancia materna en el centro-norte de España. Rev Pediatr Aten Primaria. 2007;9:589-612. 